\title{
Savaris et al (2021) erroneously interpreted their regressions
}

\author{
Carlos Góes*
}

March 2021

Savaris et al. [1] aim at "verifying if staying at home had an impact on mortality rates." This short note shows that the methodology they have applied in their paper does not allow them to do so. An estimated coefficient $\beta \approx 0$ does not imply that there is no association between the variables in either country. Rather, their pairwise difference regressions are computing coefficients that are weighted-averages of region-specific time series regressions, such that it is possible that the association is significant in both regions but their weighted-averages is close to zero. Therefore, the results do not back up the conclusions of the paper.

Consider two regions: $A$ and $B$. Suppose that the true relationships between the change in deaths per million $\left(\Delta Y_{t}^{i}\right)$ and the change in an index of staying at home $\left(\Delta X_{t}^{i}\right)$ at epidemiological week $t$ in countries $i=A, B$ are the following:

$$
\begin{aligned}
\Delta Y_{t}^{A} & =\beta_{A} \Delta X_{t}^{A}+\varepsilon_{t}^{A} \\
\Delta Y_{t}^{B} & =\beta_{B} \Delta X_{t}^{B}+\varepsilon_{t}^{B}
\end{aligned}
$$

For simplicity in exposition, assume that $X_{t}^{A}, X_{t}^{B}, \varepsilon_{t}^{A}, \varepsilon_{t}^{B}$ are all zero mean, iid processes. By subtracting the second equation from the first and defining $\Delta Y_{t} \equiv \Delta Y_{t}^{A}-\Delta Y_{t}^{B}$ and $\Delta X_{t} \equiv$ $\Delta X_{t}^{A}-\Delta X_{t}^{B}$, we can write:

$$
\begin{aligned}
\Delta Y_{t}^{A}-\Delta Y_{t}^{B} & =\beta\left(\Delta X_{t}^{A}-\Delta X_{t}^{B}\right)+\left(\beta_{A}-\beta\right) \Delta X_{t}^{A}-\left(\beta_{B}-\beta\right) \Delta X_{t}^{B}+\left(\varepsilon_{t}^{A}-\varepsilon_{t}^{B}\right) \\
\Delta Y_{t} & =\beta \Delta X_{t}+\eta_{t}
\end{aligned}
$$

where $\eta_{t} \equiv\left(\beta_{A}-\beta\right) \Delta X_{t}^{A}-\left(\beta_{B}-\beta\right) \Delta X_{t}^{B}+\left(\varepsilon_{t}^{A}-\varepsilon_{t}^{B}\right)$. It is easy to see that, for $\beta_{i} \neq \beta$, estimation of $\beta$ will not be consistent, since, by construction, $\operatorname{cov}\left(\Delta X_{t}, \eta_{t}\right) \neq 0$.

If nonetheless one estimates (1) by ordinary least squares, what does the regression coefficient $\beta$ converge to? One can calculate it analytically:

\footnotetext{
*University of California - San Diego, Department of Economics. cgoes@ucsd.edu
} 


$$
\begin{aligned}
\operatorname{plim} \beta & =\frac{\mathbb{E}\left[\Delta Y_{t} \Delta X_{t}\right]}{\mathbb{E}\left[\Delta X_{t}^{2}\right]} \\
& =\frac{\mathbb{E}\left[\left(\Delta Y_{t}^{A}-\Delta Y_{t}^{B}\right)\left(\Delta X_{t}^{A}-\Delta X_{t}^{B}\right)\right]}{\mathbb{E}\left[\left(\Delta X_{t}^{A}-\Delta X_{t}^{B}\right)^{2}\right]} \\
& =\frac{\mathbb{E}\left[\Delta Y_{t}^{A} \Delta X_{t}^{A}\right]+\mathbb{E}\left[\Delta Y_{t}^{B} \Delta X_{t}^{B}\right]}{\mathbb{E}\left[\left(\Delta X_{t}^{A}\right)^{2}\right]+\mathbb{E}\left[\left(\Delta X_{t}^{B}\right)^{2}\right]} \quad \because \mathbb{E}\left[\Delta X_{t}^{A} \Delta X_{t}^{B}\right]=\mathbb{E}\left[\Delta X_{t}^{A} \Delta Y_{t}^{B}\right]=\mathbb{E}\left[\Delta X_{t}^{B} \Delta Y_{t}^{A}\right]=0 \\
& =\frac{\mathbb{E}\left[\left(\Delta X_{t}^{A}\right)^{2}\right]}{\mathbb{E}\left[\left(\Delta X_{t}^{A}\right)^{2}\right]+\mathbb{E}\left[\left(\Delta X_{t}^{B}\right)^{2}\right]} \frac{\mathbb{E}\left[\Delta Y_{t}^{A} \Delta X_{t}^{A}\right]}{\mathbb{E}\left[\left(\Delta X_{t}^{A}\right)^{2}\right]}+\frac{\mathbb{E}\left[\left(\Delta X_{t}^{B}\right)^{2}\right]}{\mathbb{E}\left[\left(\Delta X_{t}^{A}\right)^{2}\right]+\mathbb{E}\left[\left(\Delta X_{t}^{B}\right)^{2}\right]} \frac{\mathbb{E}\left[\Delta Y_{t}^{B} \Delta X_{t}^{B}\right]}{\mathbb{E}\left[\left(\Delta X_{t}^{B}\right)^{2}\right]}
\end{aligned}
$$

Finally note that $\frac{\mathbb{E}\left[\Delta Y_{t}^{A} \Delta X_{t}^{A}\right]}{\mathbb{E}\left[\left(\Delta X_{t}^{A}\right)^{2}\right]} \stackrel{P}{\rightarrow} \beta_{A}$ and $\frac{\mathbb{E}\left[\Delta Y_{t}^{B} \Delta X_{t}^{B}\right]}{\mathbb{E}\left[\left(\Delta X_{t}^{B}\right)^{2}\right]} \stackrel{P}{\rightarrow} \beta_{B}$. Therefore, defining $w \equiv \frac{\mathbb{E}\left[\left(\Delta X_{t}^{A}\right)^{2}\right]}{\mathbb{E}\left[\left(\Delta X_{t}^{A}\right)^{2}\right]+\mathbb{E}\left[\left(\Delta X_{t}^{B}\right)^{2}\right]}$, we show that $\beta$ converges in probability to a weighted average between $\beta_{A}$ and $\beta_{B}$ :

$$
\operatorname{plim} \beta=w \beta_{A}+(1-w) \beta_{B}
$$

The intuition regarding (2) is simple. Whenever the variance of $\Delta X_{t}^{A}$ is large relative to country $B, w \rightarrow 1$ and $\beta \rightarrow \beta_{A}$. Similarly, if the variance of $\Delta X_{t}^{B}$ is large relative to country $A, w \rightarrow 0$ and $\beta \rightarrow \beta_{B}$.

What does this mean for the analysis of Savaris et al. [1]? Suppose countries $A$ and $B$ have identical variance in their independent variables, but $\beta_{A}, \beta_{B}$ are different. In country $A$, the policymaker adjusts stay-at-home orders in response to the increase in deaths, such that the change in the percentage of the public staying at home is positively correlated with the change in deaths. In country $B$, the policymaker does not act, such that the change in share of population staying at home is negatively correlated with contacts, infections, and deaths.

Consider the limiting case in which $\beta_{B}=-\beta_{A}$. Then, since the regions have identical variance, $w=1 / 2$ and $\operatorname{plim} \beta=0$ even though the true association is nonzero in both countries. The regression coefficients in Savaris et al. [1] should not lead one to conclude that, in either country, there is no association between the change in mobility and the change in deaths per million. Figure 1 shows the result of 10,000 simulated $\beta$ in which $\beta_{A}=10$ and $\beta_{B}=-10$. In this case, $\operatorname{var}\left(X_{t}^{A}\right)=\operatorname{var}\left(X_{t}^{B}\right)$ and variables are iid and normally distributed. As expected, sample estimates are distributed around the population value of $\beta=0$.

For $\beta_{A} \neq \beta_{B}$, then, region-specific dynamics are heterogeneous and, as shown by Pesaran \& Smith [2], aggregating or pooling slopes can lead to biased estimates, making individual regressions for each group member preferable. If authors assume that $\beta_{A}=\beta_{B}$ for each pair in their sample i.e., homogeneous $\beta$-, then dynamic panels would have many advantages in terms of efficiency and use of instruments to circumvent endogeneity. In either case, their pairwise approach would not be appropriate.

In order to verify if "staying at home had an impact on mortality rates," it would be necessary to address many other issues in the analysis, including, but not limited to, omitted variable bias, measurement error, and endogeneity of the regressors. However, as shown above, even in a purely correlational analysis, with no causality claims, the applied methodology will simply deliver a weighted-average of coefficients across the two regions. An estimated coefficient $\beta \approx 0$ does not imply that there is no association between the variables in either country. Therefore, their conclusion does not follow from their regressions. 


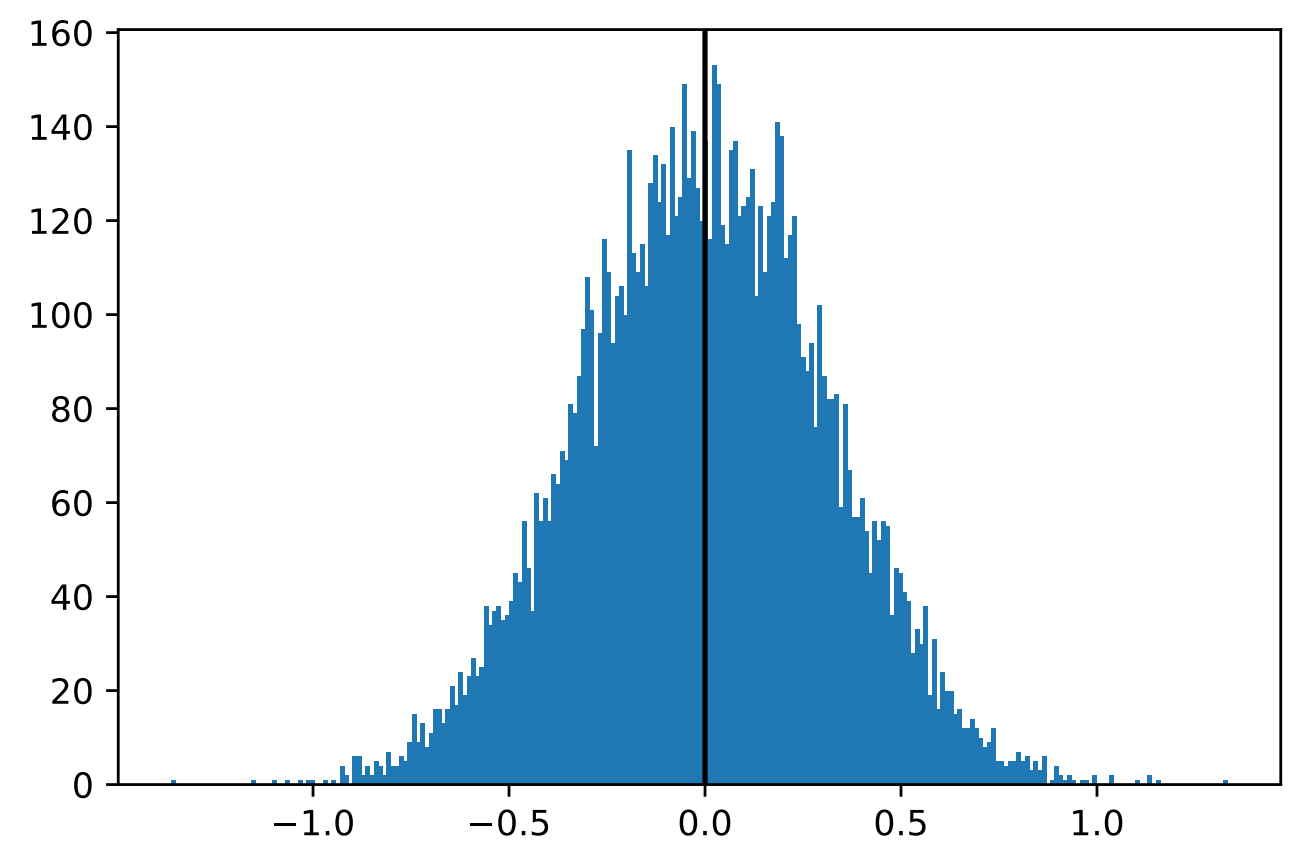

Figure 1: In-sample simulated for 10,000 random draws with $X_{t}^{i} \sim N(0,10), \varepsilon_{t}^{i} \sim N(0,1)$, and $Y_{t}^{i}=\beta_{i} X_{t}^{i}+\varepsilon_{t}^{i}$, for $i=A, B ; T=1,000 ;$ and $\beta_{A}=10, \beta_{B}=-10$. As expected the sample values are distributed around the true population value of $\beta=0$. 


\section{References}

1. Savaris, R. F., Pumi, G., Dalzochio, J. \& Kunst, R. Stay-at-home policy is a case of exception fallacy: an internet-based ecological study. Scientific Reports 11, 5313. ISSN: 2045-2322. https: //doi.org/10.1038/s41598-021-84092-1 (Mar. 2021).

2. Pesaran, M. H. \& Smith, R. Estimating long-run relationships from dynamic heterogeneous panels. Journal of Econometrics 68, 79-113 (1995).

\section{A Additional Information}

\section{A.1 Competing interests}

The author declares no competing interests.

\section{B Simulation Code}

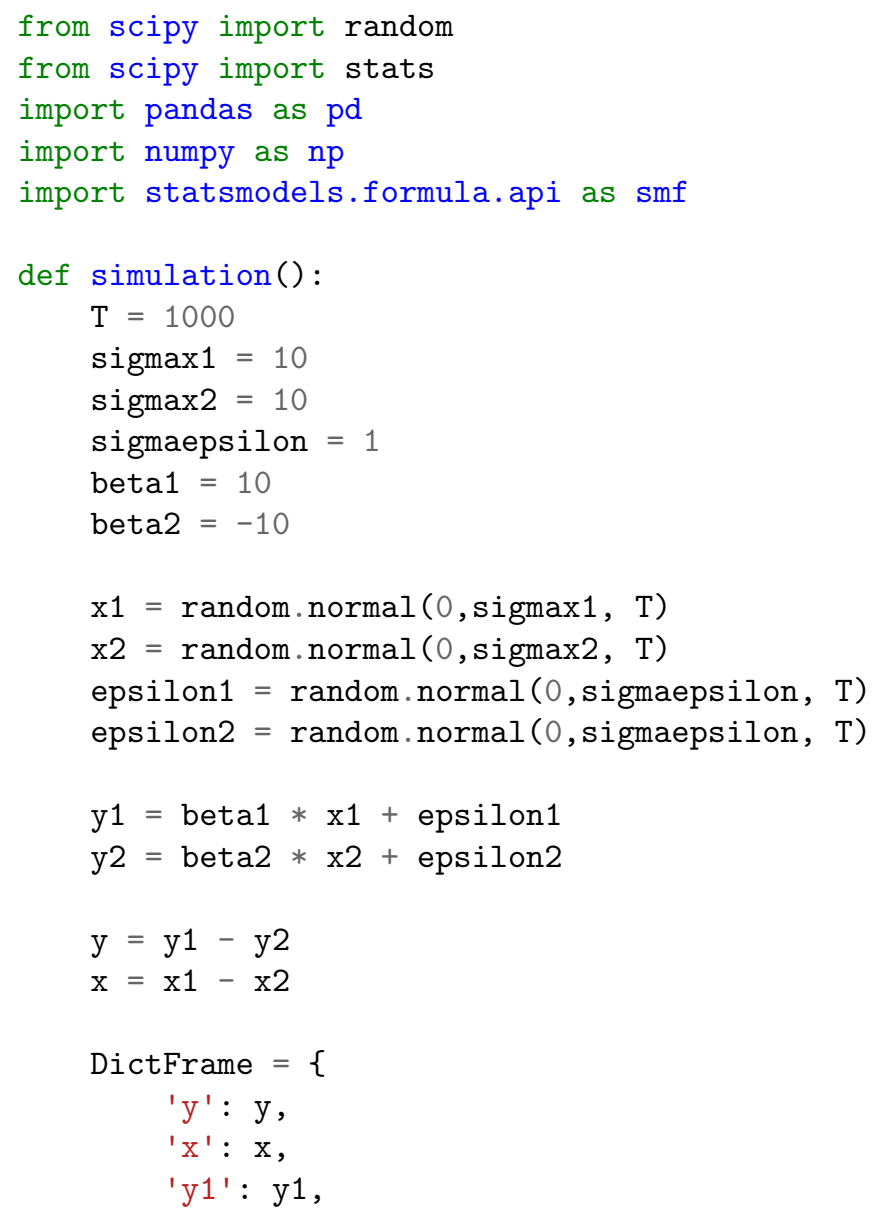




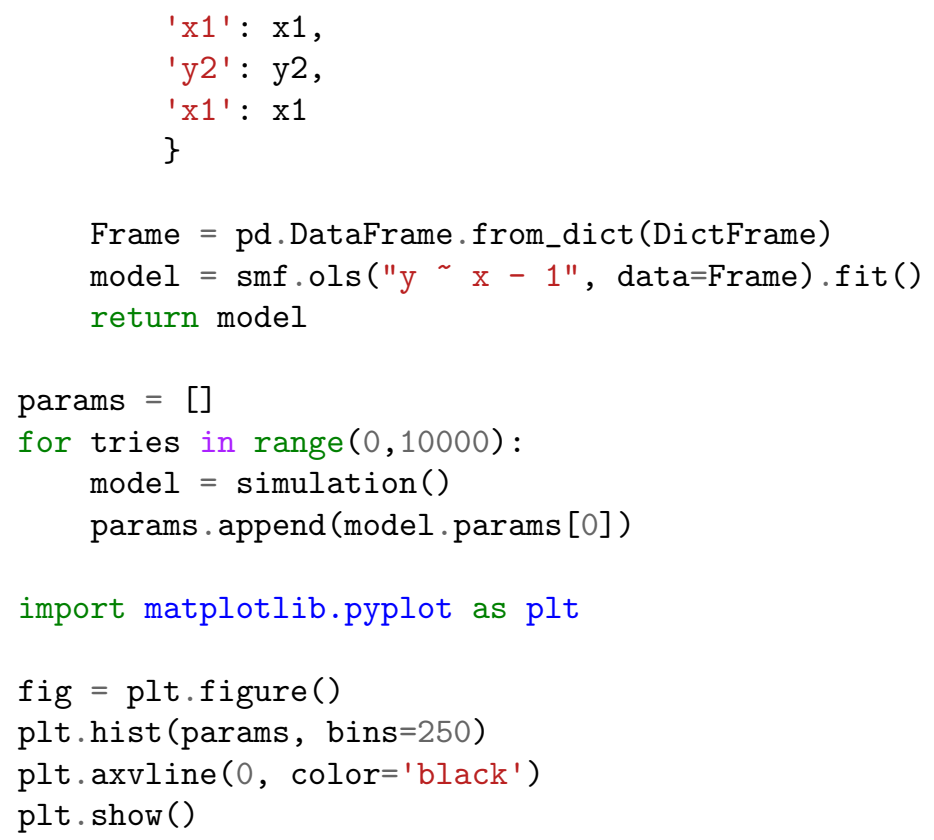

